\title{
Costos económicos de la infección respiratoria aguda en un Municipio de Colombia
}

\author{
Economic costs of acute respiratory infection \\ in a municipality of Colombia
}

Consuelo Bernal-Aguirre ${ }^{1}$; Héctor Carvajal-Sierra ${ }^{1}$; Nelson-J Alvis-Zakzuk ${ }^{1,2}$

Forma de citar: Bernal Aguirre C, Carvajal Sierra H, Alvis-Zakzuk NJ. Costos económicos de la infección respiratoria aguda en un Municipio de Colombia. Rev Univ Ind Santander Salud. 2017; 49(3): 470-477. doi: http://dx.doi.org/10.18273/revsal.v49n3-2017005 @) (1)

\section{Resumen}

Objetivo: Estimar los costos económicos de las infecciones respiratorias agudas (IRA) en el municipio de AquitaniaBoyacá, 2014. Métodos: Evaluación económica parcial, tipo descripción de costos desde la perspectiva del sistema de salud. Se utilizó la técnica de micro-costeo para estimar los costos asociados a las IRA en Aquitania-Boyacá. Los costos se discriminaron por los siguientes rubros: consultas, exámenes de laboratorio e imágenes, medicamentos, materiales e insumos y estancia hospitalaria. Se utilizaron medidas de tendencia central para resumir la información con sus respectivas medidas de dispersión. Los análisis estadísticos se realizaron en Microsoft Excel $2013 \AA$. Resultados: Los 1.576 pacientes, representaron 2.471 atenciones por IRA en la Empresa Social del Estado (ESE) Salud Aquitania, de las cuales el 15,6\% fueron en el servicio de urgencias, $81,4 \%$ en consulta externa y $3 \%$ en el servicio de hospitalización de la ESE Salud Aquitania. El promedio de estancia hospitalaria fue de 2,5 días, (máximo de cinco días). El costo promedio de un paciente con IRA hospitalizado fue \$759.437 (RIC\$639.132-\$879.742); para mujeres fue de $\$ 753.879$ (RIC\$548.862-\$877.895) y en hombres de \$764.397 (RIC\$618.523-\$910.271). El rubro que representó un mayor peso en el total del costo por caso hospitalizado fue la estancia hospitalaria, con el 64\%. Conclusión: las IRA representan una elevada carga económica al municipio de Aquitania Boyacá. La carga económica para el sistema de salud de la atención de pacientes ambulatorios en Aquitania-Boyacá en 2014 fue de \$78.9 millones de pesos y la relacionada con la hospitalización fue de \$56.198.338 (IC95\% \$ 47.295.768 $\$ 65.100 .908)$.

Palabras clave: Costos de enfermedad; Análisis de costos; Evaluación económica; Costos directos; Infección respiratoria aguda.

\footnotetext{
Abstract

Objective: To estimate the economic costs related to Acute Respiratory Infections (ARI) in a Colombian municipality. Methods: A retrospective cost-of-illness study was conducted to estimate economic costs due to ARI in Aquitania-Boyacá. The micro-costing (bottom-up) technique was used to estimate the costs associated with ARI

1. Universidad Santo Tomás. Bogotá, Colombia.

2. ALZAK Foundation. Cartagena. Bolívar, Colombia.

Correspondencia: Hector Carvajal Sierra. Dirección: Calle 65B No. 88 - 28 interior 6 apto 301 Torre campo VI. Bogotá, D.C. Colombia. Correo electrónico: hectorhernando.carvajal@gmail.com. Teléfono: +57 3214421172.
} 
in the municipality of Aquitania-Boyacá. Costs were divided by the following items: consultations, laboratory and images, drugs, materials and supplies and the hospital stay. Measures of central tendency were used to summarize the information with their respective dispersion measures. Statistical analyzes were performed in Microsoft Excel 2013®. Results: The 1,576 patients accounted for 2,471 ARI in the Empresa Social del Estado (ESE) Salud Aquitania, of which $15.6 \%$ were in the emergency department, $81.4 \%$ in the outpatient department and $3 \%$ in the hospitalization service. The average length of stay was 2.5 days (maximum of 5 days). The mean cost of a patient with hospitalized ARI was $\$ 759,437$ (ICR $\$ 639,132$ - \$ 879,742); for women was $\$ 753,879$ (ICR \$548,862- \$ 877,895 ) and for men $\$ 764,397$ (ICR \$618,523- \$910,271). Length of stay was the largest share of total cost per case, with $64 \%$. Conclusion: ARI represented a high economic burden to the municipality of Aquitania-Boyacá. The economic burden for the health care system of outpatient in the municipality in 2014 was $\$ 78,9$ million pesos and that related to hospitalization was \$56,198,338 (IC95\% \$ 47,295,768- \$65,100,908).

Keywords: Illness costs; Cost analysis; Economic evaluation; Direct costs; Acute respiratory illness.

\section{Introducción}

Las infecciones respiratorias agudas (IRA) son muy frecuentes y representan un importante problema de salud pública en el mundo ${ }^{1-5}$. Éstas, constituyen un grupo de enfermedades que se producen en el sistema respiratorio, causadas por diferentes microorganismos como virus y bacterias, que comienzan de forma repentina y duran menos de dos semanas ${ }^{1}$. La mayoría de estas infecciones, como el resfriado común, son leves. Pero dependiendo del estado general de la persona, pueden complicarse y llegar a amenazar la vida, como en el caso de las neumonías ${ }^{1}$. La población más afectada son los menores de cinco años y en este grupo de edad, la causa de la infección en el 95\% de los casos son los virus, siendo de buen pronóstico, pero un $5 \%$ puede padecer complicaciones como otitis, sinusitis y neumonía. Los principales síntomas de las IRA son: fiebre, malestar general, congestión y secreción nasal. Además, se pueden presentar síntomas como tos, dolor de garganta, expectoración y dificultad respiratoria ${ }^{1}$.

A nivel mundial, las IRA representan una alta carga económica y de enfermedad para los sistemas de salud, cada año son responsables del fallecimiento de cuatro millones de niños en todo el mundo ${ }^{6}$. En Estados Unidos (EEUU) se estimó la carga económica de infecciones virales del tracto respiratorio para 2003; se observó una carga que asciende a US\$40 mil millones (US\$17 mil millones de costos directos y US\$23 mil de costos indirectos) ${ }^{7}$. Igualmente, entre 2012-2013 se estimó también en EEUU los costos asociados con el tratamiento de IRA concluyendo que en la temporada viral los costos fueron 10 veces mayor que fuera de ésta ${ }^{8}$. Para 2012 en Canadá y Estados Unidos, la actividad de influenza continuó en aumento, según la mayoría de indicadores de influenza ${ }^{9}$. En México, el porcentaje de positividad a influenza permaneció alto y similar a semanas anteriores. Influenza A (H3) fue el virus predominante en Canadá y EEUU, mientras que influenza B fue el virus predominante en México9.

En Centro-América y el Caribe en 2012, se observó actividad elevada de IRA y aumento de detección de virus $^{10}$. A su vez, en los países de Suramérica la actividad de las IRA es alta pero con indicadores que muestran una aparente tendencia descendente, con excepción de Uruguay y el sur de Brasil, donde continúa en ascenso ${ }^{11}$. Las enfermedades del sistema respiratorio son la tercera causa de mortalidad en menores de cinco años en Suramérica ${ }^{12}$. La transición epidemiológica en América Latina y el Caribe presenta características propias que ameritan el desarrollo de modalidades de suministro de servicios más adaptadas a las realidades particulares de la región ${ }^{11}$.

En Colombia, para 2011, se reportaron más de cuatro millones de casos de IRA ${ }^{1}$. Según cifras del Instituto Nacional de Salud de Colombia, hasta la semana epidemiológica 48 de 2015 se notificaron 5.045.541 consultas externas y urgencias por IRA, 184.048 hospitalizaciones en sala general por IRA graves y 12.232 hospitalizaciones en unidad de cuidados intensivos ${ }^{13}$. La mortalidad por IRA en Colombia para 2015 fue de 12,1 por cada 100.000 menores de cinco años ${ }^{13}$.

En América Latina se han desarrollado estudios para estimar los costos de la atención por neumonía, principalmente orientados a los análisis de costoefectividad de la vacunación contra neumococo. Se ha reportado una gran variabilidad en las estimaciones de costos promedios por atención a pacientes hospitalizados por neumonía, oscilando entre US\$ 849,5 a US\$5.547, con escasa utilización de técnicas de micro-costeo para su estimación ${ }^{14-17}$. 
De acuerdo a lo anterior, y conociendo la escasez de estudios de descripción de costos de enfermedades infecciosas en Colombia, resulta importante y útil en términos de política pública valorar el consumo de recursos relacionados con las IRA. A su vez, este tipo de estudios agrega evidencia y aporta conocimiento para la toma de decisiones en el sistema de salud colombiano, especialmente a nivel municipal en hospitales de primer nivel de atención. El objetivo del presente estudio fue estimar los costos directos médicos asociados a la atención de las IRA, en un municipio del departamento de Boyacá.

\section{Métodos}

El presente estudio, es una evaluación económica parcial, tipo descripción de $\operatorname{costos}^{18}$, que estimó los costos relacionados con la atención de pacientes con IRA en Aquitania-Boyacá, para 2014. Este municipio, para 2014 tenía una población de 16.087 habitantes, según el Departamento Administrativo Nacional de Estadística (DANE). Aquitania tiene una alta carga de enfermedad asociada a enfermedades trasmisibles, según el Análisis de Situación en Salud (ASIS), realizado en $2015^{19}$. De acuerdo a éste, las IRA, representaron el $65,4 \%$ de éstas enfermedades ${ }^{19}$.

La estimación de costos de atención de IRA se llevó a cabo en la Empresa Social del Estado (ESE) Salud Aquitania, hospital de primer nivel de atención. Este centro de salud, cuenta con siete camas de hospitalización y tres de observación y presta los servicios de medicina general, consulta externa, odontología, enfermería, servicio de urgencias, transporte asistencial, psicología, nutrición, imágenes diagnosticas (rayos $\mathrm{x}$ ), hospitalización pediátrica y entre adultos e internación obstétrica. Es la institución más importante del municipio, atiende aproximadamente 55.000 consultas anuales. Para 2014 se registraron 2.471 atenciones por diagnóstico componentes de las IRA. Dentro de la clasificación del riesgo, cuenta con capacidad instalada para los siguientes servicios: sala de enfermedades respiratorias agudas, sala de rehidratación oral y sala general de procedimientos menores. El tiempo de traslado al centro de referencia de segundo nivel es de aproximadamente una hora a la ciudad de Sogamoso ${ }^{20}$.

La estimación de los costos relacionados con la atención de IRA se desarrolló de forma retrospectiva, mediante la técnica de micro-costeo o bottom $u p^{21}$. El uso de los recursos y los costos unitarios se basaron en los registros de facturación de cada paciente atendido con los siguientes diagnósticos, según la clasificación internacional de enfermedades en su versión 10 (CIE10): J00X, J011, J018, J019, J020, J029, J030, J038, J039, J040, J041, J042, J050, J069, J129, J13X, J150, J157, J159, J180, J189, J200, J209, J219, J300, J00X, J301-304, J310, J328, J329, J343, J348, J350-353, J371, J399, J42X, J441, J449- 51, J459, J690, J80X, J90X, J920, J959, J960. Se utilizó la estadística del hospital registrada en el Sistema de Información ROCKY y se evaluaron los costos incurridos por todos los pacientes atendidos por IRA en el 2014, desde el ingreso a la ESE hasta el alta del paciente. Este estudio se realizó desde la perspectiva del sistema de salud (prestador de servicios).

Los costos directos médicos relacionados con la atención de los pacientes involucraron los siguientes rubros: imágenes diagnósticas, pruebas de laboratorio, medicamentos, consultas, estancia hospitalaria, materiales e insumos, traslados. De forma primordial, este estudio estimó los costos de pacientes hospitalizados por IRA. Además, para obtener una idea general de los costos de la enfermedad, se reportaron también aquellos relacionados con la atención de pacientes en consulta externa y en el servicio de urgencias de la ESE.

Para describir los costos, se utilizaron medidas de tendencia central que permitieron resumir la información (promedio y mediana) con sus respectivas medidas de dispersión (desviación estándar [DE], intervalos de confianza [IC95\%] y rangos intercuartílicos [RIC]). Para observar si existían o no diferencias estadísticas en los costos estimados se realizó una prueba $t$-student. Los costos se presentaron en pesos colombianos de 2014. La depuración de los datos y los análisis estadísticos se realizaron en Microsoft Excel 2013®.

\section{Resultados}

En total, se analizaron 1.576 pacientes, de los cuales el $57 \%$ fueron mujeres. La edad promedio fue de 23 años $(\mathrm{DE} \pm 26,6)$ y en total se presentaron 70 diagnósticos de IRA, según la CIE10.

La Tabla 1 identifica el peso de los diagnósticos de IRA según la CIE10 y muestra que alrededor del 59\% de los casos observados se concentra en las siguientes tres patologías: rinofaringitis aguda (resfriado común), enfermedad pulmonar obstructiva crónica no especificada e infección aguda de las vías respiratorias superiores no especificada. 
Tabla 1. Porcentaje de IRA atendidas en la ESE Salud Aquitania, según diagnóstico CIE-10. 2014.

\begin{tabular}{|c|c|c|}
\hline $\begin{array}{l}\text { Código } \\
\text { CIE10 }\end{array}$ & Descripción & $\%$ \\
\hline $\mathrm{J} 00 \mathrm{X}$ & $\begin{array}{l}\text { RINOFARINGITIS AGUDA } \\
\text { (RESFRIADO COMÚN) }\end{array}$ & 35,98 \\
\hline J449 & $\begin{array}{c}\text { ENFERMEDAD PULMONAR } \\
\text { OBSTRUCTIVA CRÓNICA, NO } \\
\text { ESPECIFICADA }\end{array}$ & 12,10 \\
\hline J069 & $\begin{array}{l}\text { INFECCIÓN AGUDA DE LAS VÍAS } \\
\text { RESPIRATORIAS SUPERIORES, NO } \\
\text { ESPECIFICADA }\end{array}$ & 10,76 \\
\hline J209 & $\begin{array}{c}\text { BRONQUITIS AGUDA, NO } \\
\text { ESPECIFICADA }\end{array}$ & 10,76 \\
\hline J039 & $\begin{array}{c}\text { AMIGDALITIS AGUDA, NO } \\
\text { ESPECIFICADA }\end{array}$ & 6,11 \\
\hline J029 & $\begin{array}{l}\text { FARINGITIS AGUDA, NO } \\
\text { ESPECIFICADA }\end{array}$ & 3,84 \\
\hline $\mathrm{J} 304$ & $\begin{array}{l}\text { RINITIS ALÉRGICA, NO } \\
\text { ESPECIFICADA }\end{array}$ & 3,12 \\
\hline $\mathrm{J} 180$ & $\begin{array}{c}\text { BRONCONEUMONÍA, NO } \\
\text { ESPECIFICADA }\end{array}$ & 1,98 \\
\hline $\mathrm{J} 459$ & ASMA, NO ESPECIFICADA & 1,78 \\
\hline $\mathrm{J} 219$ & $\begin{array}{c}\text { BRONQUIOLITIS AGUDA, NO } \\
\text { ESPECIFICADA }\end{array}$ & 1,74 \\
\hline J441 & $\begin{array}{l}\text { ENFERMEDAD PULMONAR } \\
\text { OBSTRUCTIVA CRÓNICA CON } \\
\text { EXACERBACION AGUDA, NO } \\
\text { ESPECIFICADA }\end{array}$ & 1,38 \\
\hline $\mathrm{J} 303$ & OTRAS RINITIS ALÉRGICAS & 1,05 \\
\hline J040 & LARINGITIS AGUDA & 0,97 \\
\hline $\mathrm{J} 030$ & AMIGDALITIS ESTREPTOCÓCICA & 0,81 \\
\hline J019 & $\begin{array}{c}\text { SINUSITIS AGUDA, NO } \\
\text { ESPECIFICADA }\end{array}$ & 0,69 \\
\hline $\mathrm{J} 42 \mathrm{X}$ & $\begin{array}{c}\text { BRONQUITIS CRÓNICA NO } \\
\text { ESPECIFICADA }\end{array}$ & 0,61 \\
\hline $\mathrm{J} 038$ & $\begin{array}{c}\text { AMIGDALITIS AGUDA DEBIDA } \\
\text { A OTROS MICROORGANISMOS } \\
\text { ESPECIFICADOS }\end{array}$ & 0,49 \\
\hline $\mathrm{J} 310$ & RINITIS CRÓNICA & 0,40 \\
\hline $\mathrm{J} 351$ & HIPERTROFIA DE LAS AMIGDALAS & 0,40 \\
\hline \multirow[t]{2}{*}{ J018 } & OTRAS SINUSITIS AGUDAS & 0,36 \\
\hline & OTRAS & 4,65 \\
\hline
\end{tabular}

Fuente: Base de datos pacientes con IRA del hospital ESE de Aquitania.
La Tabla 2 presenta la población que sufrió IRA y fue atendida en la ESE Salud Aquitania, por sexo y grupos etarios. Se observa que el $68 \%$ de los hombres son menores de 17 años y que la mayoría de casos para ambos sexos se presentó entre los 0-11 años, con alrededor del 50\% de los casos.

Tabla 2. Distribución de los casos de IRA por sexo y grupo etario en la ESE Salud Aquitania, 2014.

\begin{tabular}{ccccccc} 
& \multicolumn{2}{c}{ Mujer } & \multicolumn{2}{c}{ Hombre } & \multicolumn{2}{c}{ Total } \\
\hline Grupo etario & $\mathbf{n}$ & $\mathbf{\%}$ & $\mathbf{n}$ & $\mathbf{\%}$ & $\mathbf{N}$ & $\mathbf{\%}$ \\
\hline$\leq 5$ años & 237 & 26,4 & 294 & 43,2 & 531 & 33,7 \\
6 - 11 años & 138 & 15,4 & 118 & 17,3 & 256 & 16,2 \\
12 - 17 años & 78 & 8,7 & 50 & 7,3 & 128 & 8,1 \\
18 - 28 años & 76 & 8,5 & 32 & 4,7 & 108 & 6,9 \\
$29-59$ años & 228 & 25,5 & 77 & 11,3 & 305 & 19,4 \\
$\geq 60$ años & 138 & 15,4 & 110 & 16,2 & 248 & 15,7 \\
Total & 895 & 100,0 & 681 & 100,0 & 1576 & 100,0 \\
\hline
\end{tabular}

Fuente: Base de datos pacientes con IRA del hospital ESE de Aquitania.

Los 1.576 pacientes, representaron 2.471 atenciones por IRA en la ESE, de las cuales el 15,6\% fueron en el servicio de urgencias, $81,4 \%$ en la consulta externa y $3 \%$ en el servicio de hospitalización de la ESE Salud Aquitania. El costo total de las atenciones de urgencias y consulta externa se presenta en la Tabla 3.

Tabla 3. Costo total de atenciones por IRA en urgencias y consulta externa de la ESE Salud Aquitania, 2014.

\begin{tabular}{lccc}
\hline \multicolumn{1}{c}{$\begin{array}{c}\text { Rubro de } \\
\text { atención }\end{array}$} & $\begin{array}{c}\text { Consulta } \\
\text { externa }\end{array}$ & Urgencias & Total \\
\hline Consultas & $\$ 49.480 .200$ & $\$ 15.590 .700$ & $\$ 65.070 .900$ \\
Traslados & $\$ 7.796 .000$ & $\$ 7.796 .000$ \\
$\begin{array}{l}\text { Materiales e } \\
\text { insumos }\end{array}$ & $\$ 849.23$ & $\$ 849.23$ \\
$\begin{array}{l}\text { Exámenes de } \\
\text { laboratorio }\end{array}$ & & $\$ 1.683 .700$ & $\$ 1.683 .700$ \\
Medicamentos & & $\$ 1.152 .069$ & $\$ 1.152 .069$ \\
$\begin{array}{l}\text { Exámenes de } \\
\text { imagen }\end{array}$ & & $\$ 2.405 .325$ & $\$ 2.405 .325$ \\
Total & $\$ 49.480 .200$ & $\$ 29.477 .024$ & $\$ 78.957 .224$ \\
\hline
\end{tabular}

N. de atenciones ambulatorias: 2012; N. de atenciones en urgencias: 385

Fuente: Base de datos pacientes con IRA del hospital ESE de Aquitania. 
La Tabla 4, analiza los costos directos médicos de los 74 pacientes hospitalizados, de los cuales $51 \%$ fueron hombres. La edad promedio de los pacientes hospitalizados fue de 32 años. El promedio de estancia hospitalaria fue de 2,5 días, con una estancia máxima de cinco días. El costo promedio de un paciente con
IRA hospitalizado fue de $\$ 759.437$ (RIC \$639.132$\$ 879.742$ ); para mujeres fue de $\$ 753.879$ (RIC $\$ 548.862-\$ 877.895$ ) y en hombres de $\$ 764.397$ (RIC \$618.523-\$910.271). No se presentaron diferencias estadísticamente significativas en el costo de hombres y mujeres $(\mathrm{P}>0,05)$.

Tabla 4. Costos directos de atención de pacientes con IRA en la ESE Salud Aquitania, 2014.

\begin{tabular}{|c|c|c|c|c|c|c|}
\hline \multicolumn{7}{|c|}{ Total } \\
\hline \multirow{2}{*}{$\begin{array}{l}\text { Rubro de atención } \\
\text { Consultas }\end{array}$} & \multirow{2}{*}{$\begin{array}{c}\text { Promedio } \\
\$ 40.285\end{array}$} & \multicolumn{2}{|c|}{ I.C. $95 \%$} & \multirow{2}{*}{$\begin{array}{c}\text { Mediana } \\
\$ 40.500\end{array}$} & \multicolumn{2}{|c|}{ R.I.C } \\
\hline & & $\$ 39.864$ & $\$ 40.706$ & & $\$ 40.500$ & $\$ 40.500$ \\
\hline Laboratorios & $\$ 32.839$ & $\$ 27.766$ & $\$ 37.912$ & $\$ 30.950$ & $\$ 17.000$ & $\$ 44.900$ \\
\hline Imágenes diagnósticas & $\$ 31.978$ & $\$ 26.119$ & $\$ 37.838$ & $\$ 36.975$ & $\$ 36.975$ & $\$ 36.975$ \\
\hline Medicamentos & $\$ 113.731$ & $\$ 90.592$ & $\$ 136.870$ & $\$ 87.355$ & $\$ 39.950$ & $\$ 160.048$ \\
\hline Materiales e insumos & $\$ 24.275$ & $\$ 20.828$ & $\$ 27.722$ & $\$ 20.850$ & $\$ 16.325$ & $\$ 32.650$ \\
\hline Traslados & $\$ 32.432$ & $\$ 15.521$ & $\$ 49.344$ & & & \\
\hline Estancia & $\$ 483.896$ & $\$ 418.442$ & $\$ 549.350$ & $\$ 468.000$ & $\$ 280.800$ & $\$ 611.775$ \\
\hline Costo directo médico & $\$ 759.437$ & $\$ 639.132$ & $\$ 879.742$ & $\$ 684.630$ & $\$ 394.575$ & $\$ 926.848$ \\
\hline \multicolumn{7}{|c|}{ Mujeres } \\
\hline Rubro de atención & Promedio & \multicolumn{2}{|c|}{ I.C. $95 \%$} & Mediana & \multicolumn{2}{|c|}{ R.I.C } \\
\hline Consultas & $\$ 40.500$ & $\$ 40.500$ & $\$ 40.500$ & $\$ 40.500$ & $\$ 40.500$ & $\$ 40.500$ \\
\hline Laboratorios & $\$ 35.603$ & $\$ 26.800$ & $\$ 44.405$ & $\$ 35.600$ & $\$ 17.000$ & $\$ 45.550$ \\
\hline Imágenes diagnósticas & $\$ 25.354$ & $\$ 16.780$ & $\$ 33.929$ & $\$ 36.975$ & $\$ 36.975$ & $\$ 36.975$ \\
\hline Medicamentos & $\$ 97.180$ & $\$ 72.939$ & $\$ 121.421$ & $\$ 76.560$ & $\$ 42.495$ & $\$ 142.100$ \\
\hline Materiales e insumos & $\$ 25.330$ & $\$ 22.747$ & $\$ 27.913$ & $\$ 24.750$ & $\$ 16.375$ & $\$ 31.950$ \\
\hline Traslados & $\$ 27.778$ & $\$ 4.863$ & $\$ 50.692$ & & & \\
\hline Estancia & $\$ 502.133$ & $\$ 404.732$ & $\$ 599.535$ & $\$ 468.000$ & $\$ 374.400$ & $\$ 578.325$ \\
\hline Costo directo médico & $\$ 753.879$ & $\$ 548.862$ & $\$ 877.895$ & $\$ 682.385$ & $\$ 490.770$ & $\$ 875.400$ \\
\hline \multicolumn{7}{|c|}{ Hombres } \\
\hline Rubro de atención & Promedio & \multicolumn{2}{|c|}{ I.C. $95 \%$} & Mediana & \multicolumn{2}{|c|}{ R.I.C } \\
\hline Consultas & $\$ 40.082$ & $\$ 39.261$ & $\$ 40.902$ & $\$ 40.500$ & $\$ 40.500$ & $\$ 40.500$ \\
\hline Laboratorios & $\$ 30.221$ & $\$ 24.932$ & $\$ 35.510$ & $\$ 22.450$ & $\$ 17.000$ & $\$ 44.900$ \\
\hline Imágenes diagnósticas & $\$ 37.948$ & $\$ 30.305$ & $\$ 45.591$ & $\$ 36.975$ & $\$ 36.975$ & $\$ 49.300$ \\
\hline Medicamentos & $\$ 129.411$ & $\$ 90.966$ & $\$ 167.857$ & $\$ 98.170$ & $\$ 39.800$ & $\$ 175.023$ \\
\hline Materiales e insumos & $\$ 23.275$ & $\$ 18.454$ & $\$ 28.096$ & $\$ 18.600$ & $\$ 15.575$ & $\$ 31.675$ \\
\hline Traslados & $\$ 36.842$ & $\$ 36.841$ & $\$ 36.843$ & & & \\
\hline Estancia & $\$ 466.618$ & $\$ 377.765$ & $\$ 555.472$ & $\$ 439.950$ & $\$ 280.800$ & $\$ 611.775$ \\
\hline Costo directo médico & $\$ 764.397$ & $\$ 618.523$ & $\$ 910.271$ & $\$ 656.645$ & $\$ 430.650$ & $\$ 953.173$ \\
\hline
\end{tabular}

Fuente: Base de datos pacientes con enfermedad IRA del hospital ESE de Aquitania. 

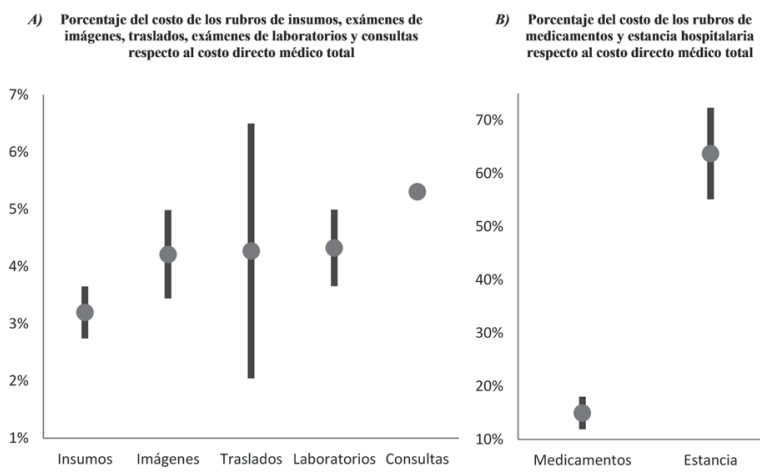
medicamentos y estancia hospitalaria
respecto al costo directo médico total

Figura 1. Distribución porcentual de los costos directos médicos por rubros de atención, 2014.

La Figura 1 resume la distribución de los rubros de atención en hospitalizados frente al total. Se evidencia que del costo directo médico total, la estancia hospitalaria representó la mayor proporción, con el 64\% (ver panel B), y el rubro de menor porcentaje fueron los materiales e insumos, con el 3\% (ver panel A).

\section{Discusión}

Según nuestro conocimiento, el presente es el primer estudio que estima los costos asociados a la atención de pacientes con IRA en el municipio de Aquitania-Boyacá.

Los estudios de descripción de costos son parte central de la evaluación económica en salud ${ }^{22,23}$. Éstos cuantifican económicamente los recursos consumidos en la prevención, tratamiento y cuidado de la enfermedad ${ }^{23,24}$. Son importantes porque estiman la cantidad de dinero que se ahorraría en ausencia de enfermedad, la cual podría ser direccionada hacia otros sectores $^{23,25}$. Además, ayudan a tomar decisiones en políticas de salud pública y son útiles porque permiten obtener los insumos necesarios para realizar futuras evaluaciones económicas completas de tecnologías sanitarias $^{23}$.

La mediana del costo estimada en este estudio para atender un caso de IRA en Aquitania (Boyacá) fue de $\$ 684.630$ (RIC \$394.575 - \$926.848), alrededor de un $11 \%$ mayor que el salario mínimo legal para 2014. En nuestro análisis se reportaron promedios y medianas de los costos, con el fin de generar evidencia suficiente para próximos estudios de eficiencia económica que necesiten como insumo la valoración de las IRA. Sin embargo, en los estudios de costos se recomienda tener en cuenta la mediana como medida resumen de los costos, debido a la variabilidad que éstos presentan y porque normalmente su distribución es asimétrica ${ }^{26}$. Se resalta que no se presentaron diferencias significativas en los costos estimados por sexo en la ESE Salud Aquitania.

La cantidad de procesos y procedimientos que demandan las IRA, tales como la enfermedad pulmonar obstructiva, rinofaringitis aguda e infección aguda de las vías respiratorias superiores no especificada, las cuales representaron para la ESE Salud Aquitania el $59 \%$ de las atenciones, congestionan la consulta externa. Además, los casos que se hospitalizan, con un promedio de estancia de 2,5 días, impactan notablemente el riesgo en salud y el presupuesto general de gastos de la ESE Salud Aquitania. Lo anterior, sugiere reevaluar los programas de promoción y prevención, atención primaria en salud y plan de intervenciones colectivas.

En un sistema de salud como el colombiano, existen muchas dificultades para obtener información sobre costos, debido a problemas relacionados con la generación de la información y la aplicación de protocolos en hospitales, especialmente aquellos de primer nivel de atención ${ }^{27}$. Las IRA son un grupo de enfermedades muy frecuentes en un país tropical como Colombia, por lo que generan una alta demanda al sistema de salud. Esto implica un problema de salud pública por el número de pacientes y la cantidad de recursos que se emplean en su tratamiento ${ }^{28}$. De acuerdo al elevado consumo de recursos y considerando de forma fundamental la información presentada referida a costos, nuestros resultados son un insumo de política pública para diagnosticar el real impacto económico de las IRA, especialmente en un contexto de país que motiven la realización y supervisión de recomendaciones preventivas por las autoridades de salud $^{29}$.

Teniendo en cuenta nuestras estimaciones, se puede asumir que la carga económica para el sistema de salud de la atención de pacientes ambulatorios en el municipio de Aquitania-Boyacá en 2014 fue de $\$ 78,9$ millones de pesos (consulta externa: $\$ 49,4$ millones; urgencias: $\$ 29,5$ millones). Y la relacionada con la hospitalización fue de $\$ 56.198 .338$ (IC95\% \$ 47.295.768 - \$65.100.908). Lo anterior, es respectivo a las atenciones para casos de IRA en la ESE Salud Aquitania.

La principal fortaleza de este estudio se refiere a su diseño. Se cree generalmente que la utilización de la metodología bottom-up es la metodología estándar de oro para el costo de los servicios hospitalarios ${ }^{30}$. La metodología es fiable porque todos los componentes relevantes de los costos se identifican y valoran al nivel más detallado ${ }^{30}$. 
El presente estudio presenta una limitación que debe tenerse en cuenta al momento de generalizar los resultados. Al realizarse el costeo en un hospital de primer nivel de atención, el costo estimado resultante podría estar subestimado, puesto que se desconoce el consumo de recursos de aquellos pacientes que se trasladan a instituciones de mayor complejidad. Lo anterior, aparte de ser una limitación del estudio, corresponde a una problemática del sistema de salud colombiano, el cual no cuenta con una historia clínica unificada. Esta fragmentación de la atención y de los expedientes clínicos, impide realizar una estimación más precisa del costo económico de las enfermedades.

Un reto para futuras investigaciones en el municipio de Aquitania, es valorar los costos directos no médicos, relacionados con el gasto de bolsillo de las familias y pacientes con IRA, tal como se ha hecho anteriormente en población de todas las edades de otros países como India $^{31} \mathrm{y}$ en pacientes pediátricos en Argentina ${ }^{32}$. A su vez, medir la pérdida de productividad asociada a este grupo de enfermedades.

\section{Agradecimientos}

Los autores agradecen a las autoridades de la ESE Salud Aquitania, por el suministro de la información necesaria para la realización de este estudio.

El presente artículo es resultado de una investigación desarrollada por estudiantes de la especialización de Gerencia de Instituciones de Seguridad Social en Salud de la Universidad Santo Tomas (Bogotá, D.C).

\section{Consideraciones éticas}

Ningún dato que identifique a los participantes fue publicado ni divulgado. Este estudio se clasifica como una investigación sin riesgo para los pacientes, según la Resolución No. 8430 de 1993 del Ministerio de Salud.

\section{Conflictos de interés}

Los autores declaran no tener conflictos de interés.

\section{Referencias}

1. Ministerio de Salud y Protección Social. Infecciones Respiratorias Agudas (IRA).

2. Chocontá-Piraquive LA, Alvis Guzmán N, De la Hoz Restrepo F. Cost-effectiveness of vaccinating pregnant women against pandemic influenza in
Colombia. Rev Panam Salud Pública 2012; 31(6): 447-453.

3. Nicholson KG, Wood JM, Zambon M, Turner D, Wailoo A, Nicholson K, et al. Influenza. Lancet. 2003; 362(9397): 1733-1745.

4. Kuri-Morales P, Galván F, Cravioto P, Rosas LAZ, Tapia-Conyer R. Mortalidad en México por influenza y neumonía (1990-2005). Salud Publica Mex. 2006; 48(5): 379-384. DOI: http://dx.doi. org/10.1590/S0036-36342006000500004.

5. Bonvehí PE, Istúriz RE, Labarca JA, Rüttimann RW, Vidal EI, Vilar-Compte D. Influenza among adults in Latin America, current status, and future directions: a consensus statement. Rev Panam Salud Pública. 2012; 31(6): 506-512.

6. Pio A. Acute respiratory infections in children in developing countries: an international point of view. Pediatr Infect Dis. 1986. 5(2): 179-183.

7. Fendrick AM, Monto AS, Nightengale B, Sarnes M. The economic burden of non-influenza-related viral respiratory tract infection in the United States. Arch Intern Med. 2003; 163(4): 487-494.

8. Murray MT, Heitkemper E, Jackson O, Neu N, Stone $\mathrm{P}$, Cohen B, et al. Direct costs of acute respiratory infections in a pediatric long-term care facility. Influenza Other Respi Viruses. 2016; 10(1): 34-36.

9. Organización Panamericana de la Salud - OPS. Actualización regional Influenza y otros virus respiratorios, semana 29 de 2013. Ginebra: OPS. 2013.

10. Organización Panamericana de la Salud. Actualización Regional SE 11 , 2012. 2012;1-10.

11. Frenk J, Frejka T, Bobadilla J, Stern C. La Transición Epidemiologica en America Latina. Bol Oficina Sanit. 1991.

12. Zulma AO, Matilde BA, Lydia O, Claudia M, Patricia S, Jorge U, et al. Estado de conocimiento y agenda de prioridades para la toma de decisiones en Infecciones Respiratorias Agudas Bajas en niños menores de 5 años de edad en Argentina. Res Ejecutivo. 2006; 7: 1-2.

13. Instituto Nacional de Salud. Infección Respiratoria Aguda (IRA). Protocolo de Vigilancia en Salud Pública. Versión 05. 2016.

14. Constenla D, Evaluating the costs of pneumococcal disease in selected Latin American countries. Rev Panam Salud Publica. 2007; 22(4): 268-278.

15. Vespa G, Constenla DO, Pepe C, Safadi MA, Berezin E, de Moraes JC, et al. Estimating the cost-effectiveness of pneumococcal conjugate vaccination in Brazil. Rev Panam Salud Publica. 2009; 26(6): 518-528.

16. Augustovski FA, García Martí S, Pichon-Riviere A, 
Debbag R. Childhood pneumococcal disease burden in Argentina. Rev Panam Salud Publica. 2009; 25(5): 423-430.

17. Castañeda-Orjuela C, Alvis-Guzmán N, Paternina AJ, De la Hoz-Restrepo F. Cost-effectiveness of the introduction of the pneumococcal polysaccharide vaccine in elderly Colombian population. Vaccine. 2011; 29(44): 7644-7650.

18. Drummond MF, Sculpher MJ, Claxton K, Stoddart GL, Torrance GW. Methods for the economic evaluation of health care programmes. Oxford university press; 2015.

19. ESE Salud Aquitania - Área de Vigilancia en Salud Pública. Análisis de situación de salud con el modelo de los determinantes sociales de salud, AquitaniaBoyacá. 2015. 2016.

20. ESE Salud Aquitania. Portafolio de servicios definitivo; 2014.

21. Frick KD. Microcosting quantity data collection methods. Med Care. 2009; 47 (7 Suppl 1): S76-81.

22. Lenz-Alcayaga R. Análisis de costos en evaluaciones económicas en salud: Aspectos introductorios. Rev Med Chil. 2010; 138: 88-92.

23. Instituto Nacional de Salud - Observatorio Nacional de Salud. Informe Nacional de las Desigualdades Sociales en Salud en Colombia. Imprenta Nac Colomb Bogotá, DC. 2015; P. 213.

24. Rice DP. Estimating the Cost-of-Illness. Washington, DC: US Department of Health, Education, and Welfare, Public Health Service. 1966.

25. Soumahoro MK, Boelle PY, Gaüzere BA, Atsou K, Pelat C, Lambert B, et al. The Chikungunya Epidemic on La Réunion Island in 2005-2006: a cost-of-illness study. Powers AM, editor. PLoS Negl Trop Dis. 2011; 5(6): 197.

26. Guerrero R, Guevara C, Parody E. Guía metodológica para la realización de evaluaciones económicas en el marco de Guías de Práctica Clínica. PROESA. 2014; 1-127.

27. Guzmán NA, De La Hoz Restrepo F, Higuera AB, Pastor D, Luis J, Fabio D. Costos económicos de las neumonías en niños menores de 2 años de edad, en Colombia. Rev Panam Salud Publica. 2005; 17(3): 178-183.

28. Garcia CM, Suarez EP. Caracterización epidemiológica de la infección respiratoria aguda grave y circulación viral en Boyacá, 2012 - 2013$2014 ; 85$.

29. Delpiano L, Kabalán P, Diaz P, Pinto A. Características $\mathrm{y}$ costos directos de infecciones respiratorias agudas en niños de guarderías infantiles. Rev Chil infectología. 2006; 23(2): 128-133.

30. Tan SS, Rutten FFH, Van Ineveld BM, Redekop WK,
Hakkaart-van Roijen L. Comparing methodologies for the cost estimation of hospital services. Eur J Heal Econ. 2009; 10(1): 39-45.

31. Peasah SK, Purakayastha DR, Koul PA, Dawood FS, Saha S, Amarchand R, et al. The cost of acute respiratory infections in Northern India: a multi-site study. BMC Public Health. 2015; 15(1): 330.

32. Rowensztein H, Demirdjian G, Rodríguez J. Carga de enfermedad y costos asociados a las internaciones por infección respiratoria aguda en niños. Arch Argent Pediatr. 2007; 105(1): 5-11. 